\title{
Altered organization of the visual cortex in FHONDA syndrome
}

\author{
Khazar Ahmadi ${ }^{a}$, Alessio Fracasso ${ }^{\text {b,c,d,e }}$, Jelle A. van Dijk ${ }^{\text {b,d }}$, Charlotte Kruijt ${ }^{f, g}$, \\ Maria van Genderen ${ }^{\mathrm{f}, \mathrm{h}}$, Serge O. Dumoulin ${ }^{\mathrm{b}, \mathrm{d}, \mathrm{i}, 1}$, Michael B. Hoffmann ${ }^{\mathrm{a}, \mathrm{j}, \text {, , } 1}$

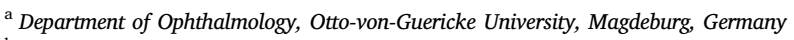 \\ ${ }^{\mathrm{b}}$ Department of Experimental Psychology, Helmholtz Institute, Utrecht University, Utrecht, The Netherlands \\ ${ }^{\mathrm{c}}$ Department of Radiology, University Medical Center Utrecht, Utrecht, The Netherlands \\ ${ }^{\mathrm{d}}$ Spinoza Centre for Neuroimaging, Amsterdam, The Netherlands \\ e Institute of Neuroscience and Psychology, University of Glasgow, Glasgow, G12 8QB, UK \\ ${ }^{\mathrm{f}}$ Bartiméus Diagnostic Center for Rare Visual Disorders, Zeist, The Netherlands \\ ${ }^{g}$ Department of Ophthalmology, Leiden University Medical Center, Leiden, The Netherlands

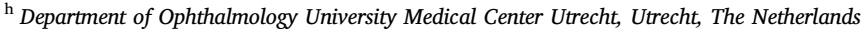 \\ ${ }^{i}$ Department of Experimental and Applied Psychology, VU University Amsterdam, Amsterdam, The Netherlands \\ ${ }^{\mathrm{j}}$ Center for Behavioral Brain Sciences, Magdeburg, Germany
}

\section{A R T I C L E I N F O}

\section{Keywords:}

FHONDA

fMRI

pRF

Plasticity

Visual cortex

\begin{abstract}
A B S T R A C T
A fundamental scheme in the organization of the early visual cortex is the retinotopic representation of the contralateral visual hemifield on each hemisphere. We determined the cortical organization in a novel congenital visual pathway disorder, FHONDA-syndrome, where the axons from the temporal retina abnormally cross to the contralateral hemisphere. Using ultra-high field fMRI at $7 \mathrm{~T}$, the population receptive field (pRF) properties of the primary visual cortex were modeled for two affected individuals and two controls. The cortical activation in FHONDA was confined to the hemisphere contralateral to the stimulated eye. Each cortical location was found to contain a pRF in each visual hemifeld and opposing hemifields were represented as retinotopic cortical overlays of mirror-symmetrical locations across the vertical meridian. Since, the enhanced crossing of the retinal fibers at the optic chiasm observed in FHONDA has been previously assumed to be exclusive to the pigment-deficiency in albinism, our direct evidence of abnormal mapping in FHONDA highlights the independence of pigmentation and development of the visual cortex. These findings thus provide fundamental insights into the developmental mechanisms of the human visual system and underline the general relevance of the interplay of subcortical stability and cortical plasticity.
\end{abstract}

\section{Introduction}

Systematic topographic representations of the visual field are a dominant feature of the visual system and thought to be essential for its functionality. They result from the interplay of pre-programmed and experience-dependent developmental mechanisms that shape the retinocortical projections (Hoffmann \& Dumoulin, 2015). Investigating the consequences of changes to these connections provides key-insights into the developmental principles of the visual system and the scope of plasticity to preserve normal functioning. While in humans experimental changes cannot be applied, patient groups have the potential to provide these profound insights.

Normally only the axons from the nasal retina project through the optic chiasm to the contralateral hemisphere while axons from the temporal retina remain ipsilateral. Consequently, the left visual hemifield is represented on the right hemisphere and vice versa. In patients with congenital malformations of the optic chiasm there can be reduced crossing of nasal axons, as reported for achiasma and hemihydranencephaly (Apkarian et al., 1994; Victor et al., 2000; Muckli et al., 2009; Hoffmann et al., 2012; Fracasso et al., 2016), or additional crossing from the temporal axons, as evident for albinism. In fact, the latter has been regarded to be pathognomonic, i.e. a unique identifier, for albinism (Apkarian et al., 1983; von dem Hagen et al., 2008). The recent discovery of the FHONDA syndrome (Al-Araimi et al., 2013; Poulter et al., 2013) clearly challenged this view and put the definition of albinism into question.

\footnotetext{
* Corresponding author. Department of Ophthalmology, Otto-von-Guericke University, Leipziger Str.44, 39120, Magdeburg, Germany.

E-mail address: Michael.hoffmann@med.ovgu.de (M.B. Hoffmann).

1 These authors contributed equally to this work.
} 
FHONDA (foveal hypoplasia, optic nerve decussation defects and anterior segment dysgenesis) is a rare inherited syndrome distinct from albinism - less than 20 individuals have been identified in the literature to-date (Al-Araimi et al., 2013; Poulter et al., 2013) -, which is caused by a recessive mutation in the putative glutamine transporter gene SLC38A8. Clinical characteristics are foveal hypoplasia - i.e., a missing foveal pit, abnormal foveal or macular reflexes and a poorly defined foveal avascular zone - and in some cases anterior segment abnormalities such as Axenfeld's anomaly or posterior embryotoxon (van Genderen et al., 2006; Al-Araimi et al., 2013; Poulter et al., 2013). Furthermore, visual evoked potentials indicate abnormal cortical response lateralization, which suggest an enhanced crossing of the retinal axons at the optic chiasm. Taken together, FHONDA shares some symptoms with albinism, i.e. the foveal hypoplasia and the optic nerve decussation defects, but in the absence of any hypopigmentation and iris translucency, which clearly distinguishes FHONDA from albinism.

As a consequence of the misrouting of the optic nerves in albinism, hemihydranencephaly, and achiasma the occipital cortex receives input not only from the contra- but also from the ipsilateral visual hemifield. This leads to substantially atypical visual field representations in the visual cortex, specifically overlaid retinotopic maps of opposing visual hemifields (Hoffmann et al., 2003; Muckli et al., 2009; Davies-Thompson et al., 2013; Kaule et al., 2014; Bao et al., 2015; Fracasso et al., 2016), as demonstrated with fMRI and eventually leading to the concept of bilateral population receptive fields (Hoffmann et al., 2012; Hoffmann \& Dumoulin, 2015). Despite this large-scale abnormal input to the visual cortex, main aspects of visual function are preserved, except for the presence of nystagmus and the reduction of binocular vision (Hoffmann et al., 2007, 2015; Klemen et al., 2012). This is taken as evidence for efficient adaptive mechanisms that make the abnormal visual input available for visual perception, and highlights the importance of these conditions as models to study mechanisms of visual system plasticity directly in humans.

The discovery of FHONDA offers a unique opportunity to test whether the previously observed interplay of subcortical stability and cortical plasticity in the human visual pathways is generally valid. The aim of the current study was to identify the projection abnormality using fMRIbased population receptive field (pRF) mapping at $7 \mathrm{~T}$. Comparing different pRF-models we determined the cortical layout of the visual field maps in two individuals with FHONDA. We provide direct evidence for large-scale abnormal input to the visual cortex and demonstrate that, despite its distinction from all other previously examined conditions with chiasmatic malformations, the cortical mapping in FHONDA follows the same principles as in human albinism, achiasma and hemihydranencephaly.

\section{Methods}

\section{Participants}

Two individuals with FHONDA [F1 and F2; age: 27 and 33, respectively; decimal visual acuity (left|right): $0.16 \mid 0.10$ and $0.20 \mid 0.16$, respectively; nystagmus amplitude of $0.65^{\circ}$ (horizontal) and $0.55^{\circ}$ (vertical) in F1 (dominant eye: right), no data available in F2 (dominant eye: left); both females] and two controls (C1 and C2, aged 32 and 23 respectively; 1 female) with no neurological and ophthalmological history and normal visual acuity took part in this study. The FHONDA participants were sisters described previously (van Genderen et al., 2006; Al-Araimi et al., 2013; Poulter et al., 2013). All participants gave their informed written consent. The protocol was approved by the ethical committee of the University Medical Center Utrecht and the procedure adhered to the tenets of the declaration of Helsinki.

\section{Visual stimulation}

For fMRI-based population receptive field mapping, drifting bar apertures exposing a moving high-contrast checkerboard pattern (Dumoulin \& Wandell, 2008) were used for four different directions. Each pass of the bars lasted for $30 \mathrm{~s}$, followed by a mean luminance block (zero contrast) of $30 \mathrm{~s}$. The stimuli were generated in Matlab (Mathworks, Natick, MA, USA) using the Psychtoolbox (Brainard, 1997; Pelli, 1997) and projected onto a screen at a resolution of $1024 \times 538$ pixels at the magnet bore. The stimulus radius was $6^{\circ}$ of visual angle. The participants viewed the screen at a distance of $35 \mathrm{~cm}$ via an angled mirror and their dominant eye was stimulated under three experimental conditions: (i) bilateral, (ii) left, and (iii) right hemifield stimulation (i.e., $0 \%$ stimulus contrast for the right and left hemifield in (ii) and (iii), respectively). Participants were required to fixate a centered dot and to report colour changes between red and green via button press. An illustration of the bilateral stimuli is exhibited in Fig. 1A. As the FHONDA-participants had

A)

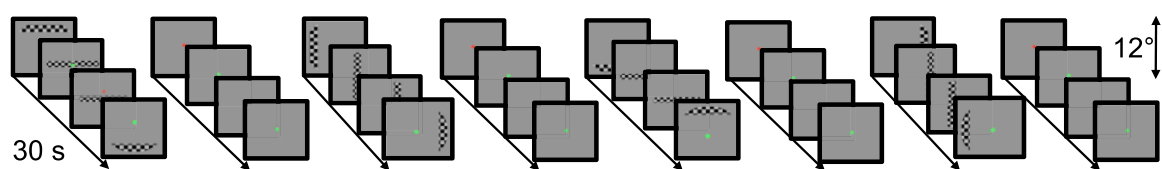

B)

(i)

single-pRF model

visual field
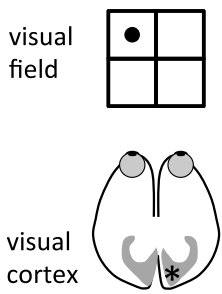

(ii)

mirror-pRF

model across VM
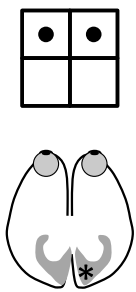

(iii)

mirror-pRF

model

across HM
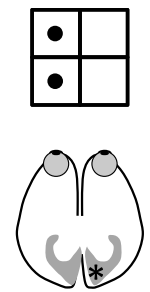

Fig. 1. A) Schematic of the visual stimulation sequence for the bilateral pRF mapping: Each column depicts screen shots of the stimulus for four successions of $30 \mathrm{~s}$ blocks of stimulation (bars moving in one of four different directions during each block) and $30 \mathrm{~s}$ fixation. For further details see Methods. B) Schematic of four pRF models: A schematic of the visual field quadrants around fixation indicates the $\mathrm{pRF}(\mathrm{s})$ in the visual field ('•') for a given location in the primary visual cortex ('*') for four different pRF models (as detailed in Methods): (i) Single-pRF model. This is the conventional model where a cortical location represents a location in the contralateral visual hemifield. (ii-iv) Mirror-pRF models are unconventional models - only (ii) has been described before (Hoffmann et al. 2012; Fracasso et al., 2016), (iii-iv) are theoretically possible, but have not been reported. (ii) Mirror-pRF model across VM (vertical meridian). A cortical location represents mirror-symmetrical locations in left and right hemifields. (iii) Mirror-pRF model across HM (horizontal meridian). A cortical location represents mirror-symmetrical locations in upper and lower contralateral hemifields. (iv) Mirror-pRF model across fixation. A cortical location represents mirror-symmetrical locations in e.g. in left upper and right lower hemifields. 

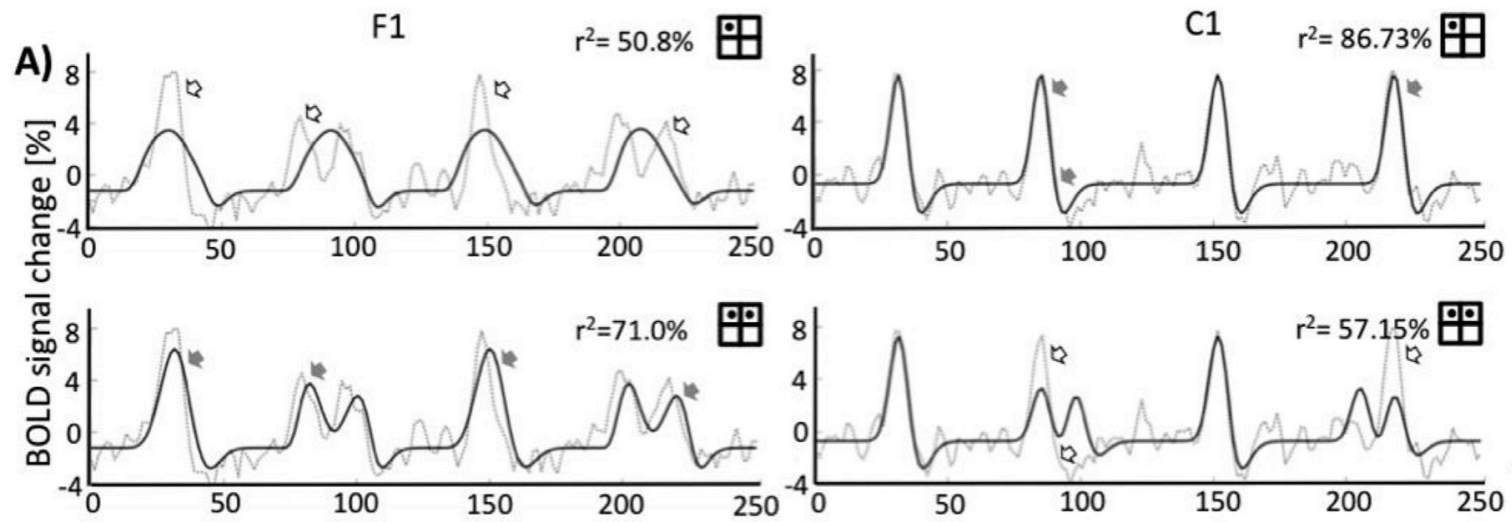

B)

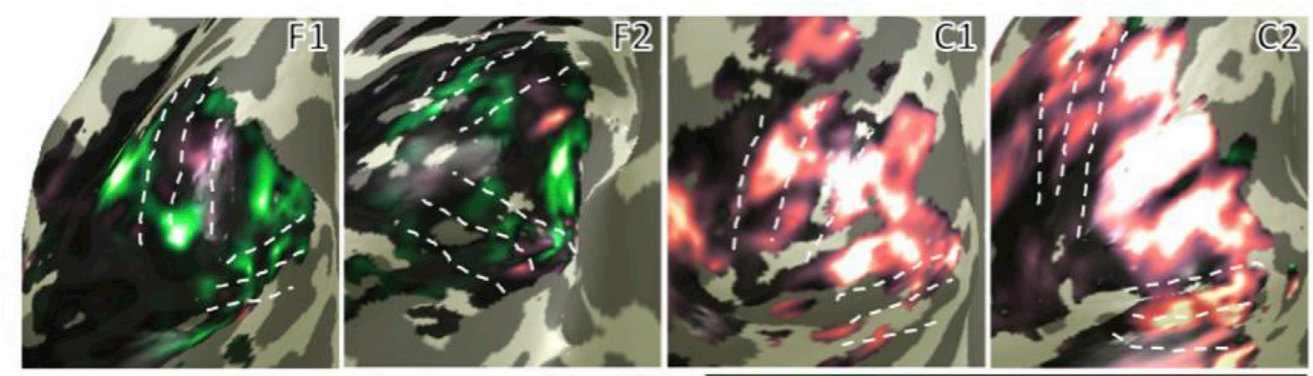

differential variance explained [\%]:
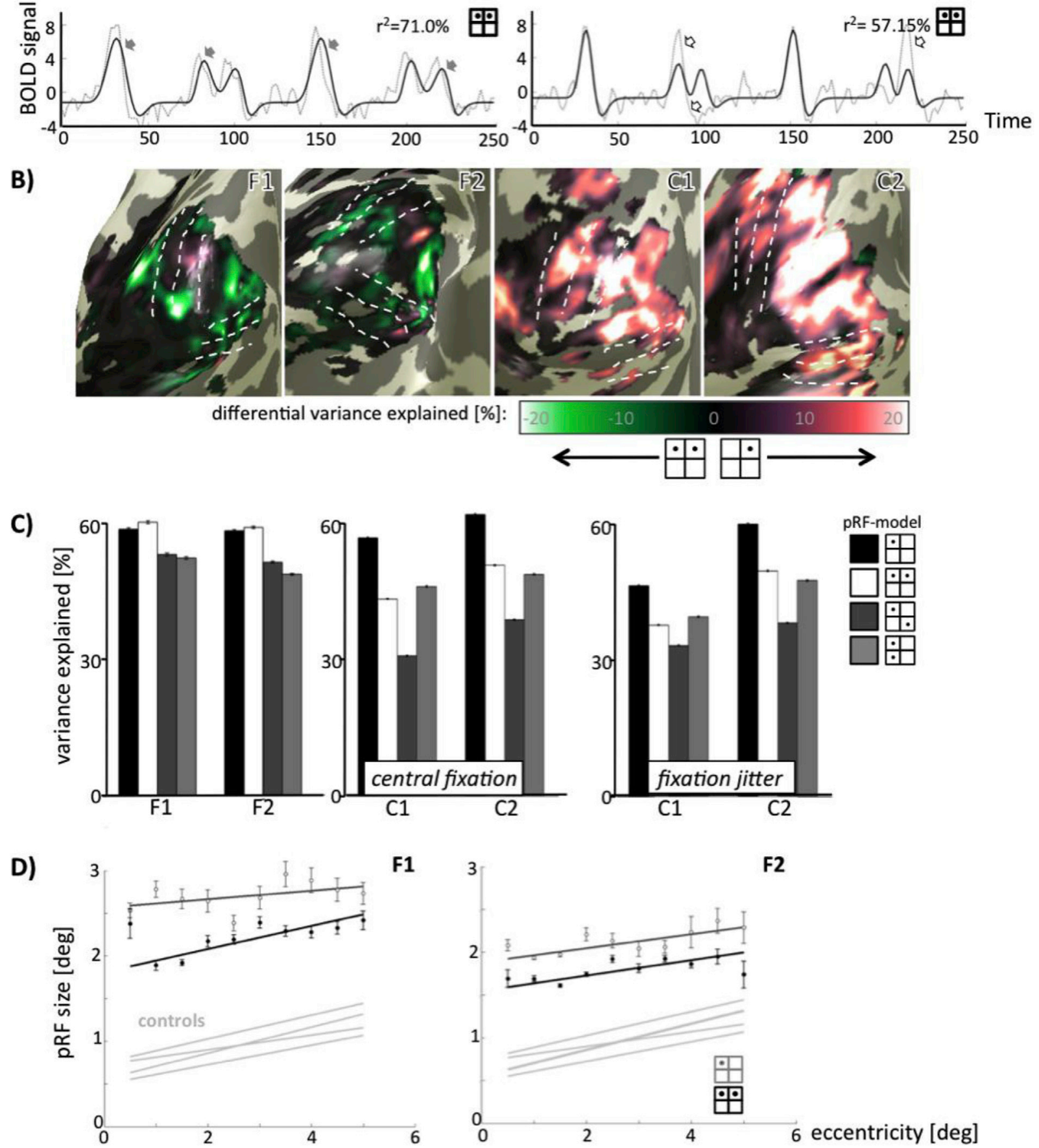

F2

6 eccentricity [deg] 


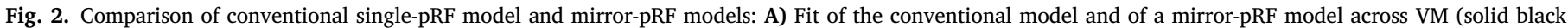

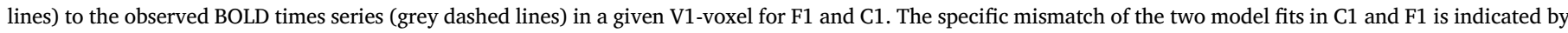

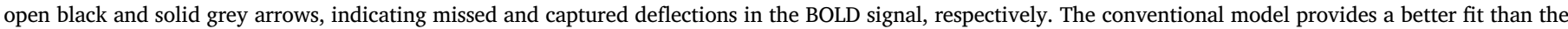

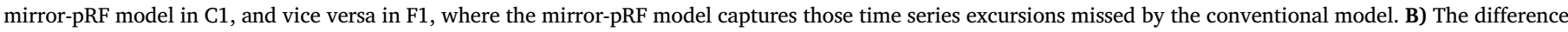

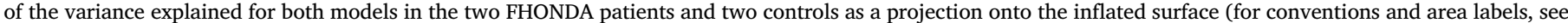

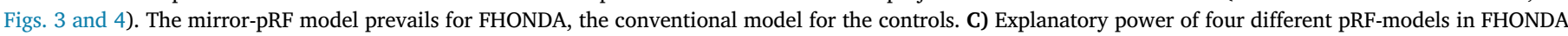

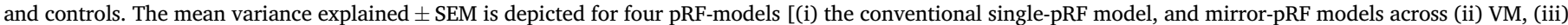

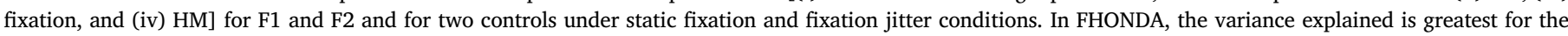

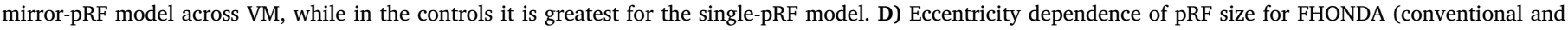

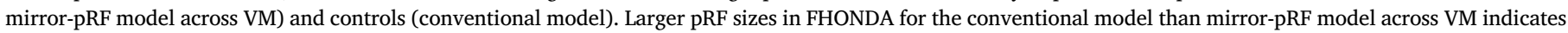
that the inadequacy of the former model is compensated for by increase in pRF size.

nystagmus, an additional stimulation condition ('fixation jitter') was introduced for the controls with the fixation dot moving in the same way as recorded for the nystagmus in F1 with an Eyelink II system (SR Research, https://www.sr-research.com/) upon observing pRF-mapping stimulus outside of the MR-scanner.

\section{MRI acquisition}

For fMRI T2*-weighted images were acquired using a multi-slice EPI sequence (TR/TE $1500 / 30 \mathrm{~ms}$, flip angle: $70^{\circ}$, voxel size: $2 \mathrm{~mm}^{3}$ isotropic and 25 coronal slices) at a 7T Achieva Philips MRI-scanner (Best, Netherlands) with a 32-channel head coil (Nova Medical, MA, USA). Each functional run had 168 time frames, lasting for a total of $252 \mathrm{~s}$. Foam padding was used to minimize head motion. Inhomogeneity corrected T1-weighted image was acquired with (TR/TE $7 / 2.84 \mathrm{~ms}$, flip angle: $8^{\circ}$ and voxel size: $0.8 \mathrm{~mm}^{3}$ ). Each left and right hemifield stimulation conditions were performed once for all of the participants. Bilateral stimulation was repeated four times in F1 and three times in F2. In controls, bilateral stimulation was divided in two parts: central fixation and fixation jitter. In C1, two repetitions with central fixation and two runs with fixation jitter were obtained. In C2, three repetitions with central fixation and two runs with fixation jitter were performed.

\section{Data analysis}

FSL (https://www.fmrib.ox.ac.uk/fsl) was used for motion correction of the functional data and segmentation of the anatomical images. Grey and white matter were further segmented by a custom software and hand edited to minimize the error (Teo et al., 1997). Motion corrected images for the same conditions were averaged together for each participant to increase the signal-to-noise (SNR) ratio. Afterwards, the anatomical image was aligned to the functional scans using mrVista (https://github. $\mathrm{com} / \mathrm{vistalab} /$ vistasoft). Due to technical issues associated with the big size of the sinus close to the right occipital cortex in $\mathrm{C} 2$, the anatomical image in this participant was co-registered to the functional runs using the 3dAllineate function of AFNI (https://afni.nimh.nih.gov/). The estimation of the population receptive field (pRF) properties of each voxel, the subsequent derivation of the polar angle and eccentricity maps for the delineation of the visual areas, and the visualization on the smoothed inflated cortical surface based on T1- weighted images were performed in mrVista. Population receptive field sizes and positions were estimated from the fMRI data and stimulus position time course. The BOLD (blood oxygen level dependent) response of each voxel was predicted using a 2D-Gaussian model of the neuronal populations defined by three stimulus-referred parameters $x_{0}, y_{0}, \sigma$ where $x_{0}$ and $y_{0}$ are the coordinates of the receptive field centre and $\sigma$ is it's spread (Dumoulin \& Wandell, 2008; Harvey \& Dumoulin, 2011; Fracasso et al., 2016). The predicted BOLD signal was calculated by convolution of the stimulus sequence for the respective pRF-model and its three parameters with the canonical hemodynamic response function (Friston et al., 1998). The pRF parameters were determined by minimizing the sum of squared errors between predicted and observed BOLD signal. For all subsequent analysis, only the voxels were included that at least $20 \%$ of their variance was explained by pRF model. To investigate whether a specific cortical region comprises conventional single or instead bilateral pRF, we extended the conventional pRF model (Hoffmann et al., 2012; Fracasso et al., 2016) as described in Fig. 1B. While the conventional model (here called 'single-pRF model') consists of a single symmetric 2D-Gaussian, the extended models (here called mirror-pRF models) comprise two mirrored 2D-Gaussians. In analogy to (Hoffmann et al., 2012; Fracasso et al., 2016) three different mirror-pRF models were used, i.e. with mirror-symmetrical pRF-locations across (i) the vertical meridian ('mirror-pRF model across VM'), (ii) the horizontal meridian ('mirror-pRF model across HM'), and (iii) fixation ('mirror-pRF model across fixation') (see Fig. 1B). In contrast to the conventional model, the three extended models predict that each cortical location responds to two distinct points in the visual field. We compared these four pRF models based on the average goodness of fit, i.e. variance explained (VE) within the primary visual cortex. Because all parameters of the two Gaussians are linked, these new models have the same degrees of freedom as the conventional single Gaussian pRF model. As a consequence the model performance can be compared directly, which would have been complicated considerably for less constrained pRF models.

\section{Results}

A first clue to the nature of the visual field mapping in the visual cortex in FHONDA was provided by the analysis of data obtained for bilateral pRF-mapping (Fig. 1A). This approach addressed the question, which pRF model prevailed in FHONDA. Specifically we compared the single-pRF model, which is adequate in controls, to mirror-pRF models, which are superior for superimposed representations of opposing hemifields (see Fig. 1B and Methods). From these analyses, depicted in Fig. 2, it was evident that the mapping in FHONDA indeed critically differed from that in controls, i.e. the results supported the notion of an overlaid representation of opposing hemifields in FHONDA. For a qualitative assessment, in Fig. 2A the BOLD time-series for a given voxel are depicted together with the model fit of two different pRF models, single- and mirror-pRF model across VM. In fact, while the former explained the control time series better, the latter explained the FHONDA time series better. This was reflected by the match of the model fits with the BOLD time series and quantitatively captured with the variance explained by the different models [VE for single and mirror-pRF models in C1: $86.73 \%$ and 57.15\%; VE for single and mirror-pRF models in F1: 50.8\% and 71\% respectively]. It should be noted that for the sake of better visualization of the model differences, we selected voxels representing an extreme difference between the two models in both participants. This discrepancy between the FHONDA and control participants is further supported by Fig. 2B, where the cortical topography of the differential variance explained (single- minus mirror-pRF model) is depicted for both subject groups. In the FHONDA participants the mirror-pRF model across VM prevailed, while the conventional single-pRF model prevailed in the controls. This was further confirmed by another analysis, a formal quantitative assessment of the mean variance explained for (i) single-pRF model and mirrored-pRF models across (ii) VM, (iii) fixation and (iv) HM depicted in Fig. 2C. This demonstrated the superiority of the mirror-pRF model across VM in FHONDA [mean VE \pm SEM for the above mentioned models (i-iv) in F1: (i) $58.74 \% \pm 0.31$, (ii) $60.32 \% \pm 0.30$, (iii) 
F1
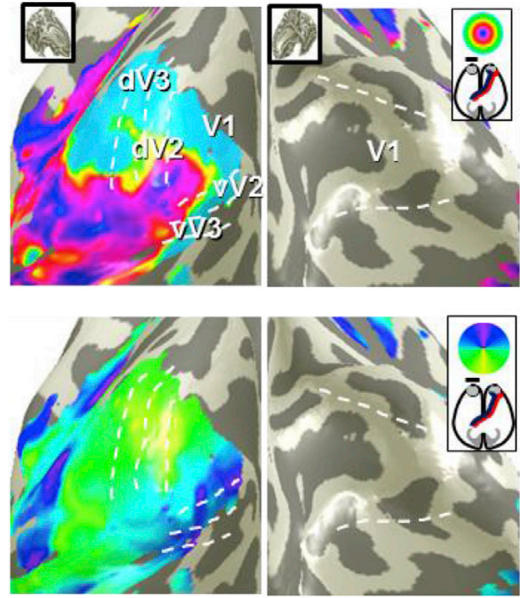

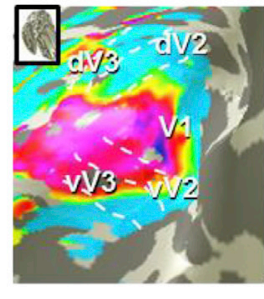

F2
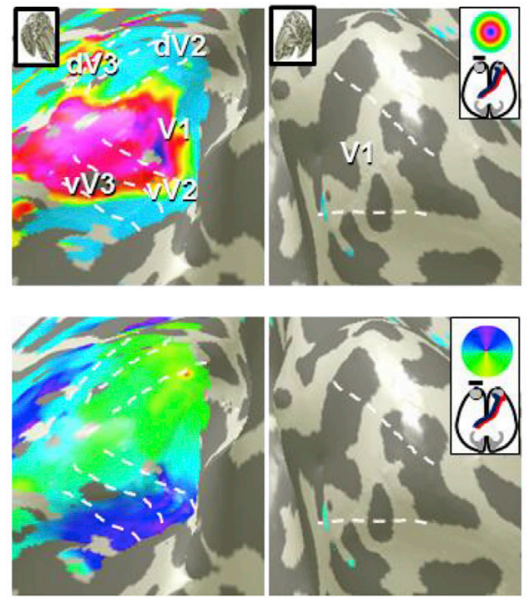

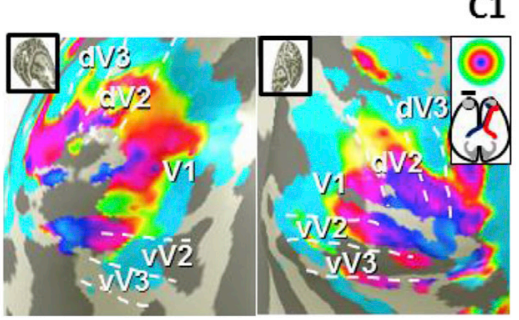

C1

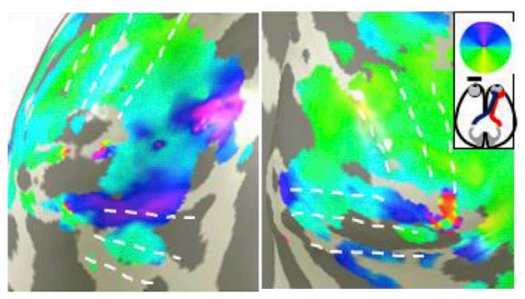

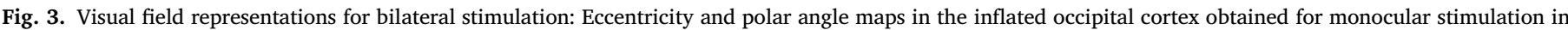

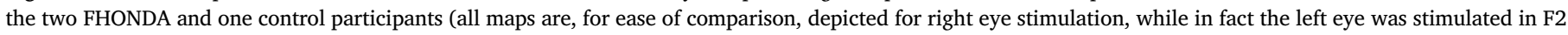

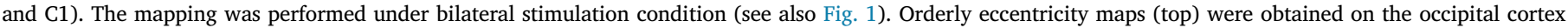

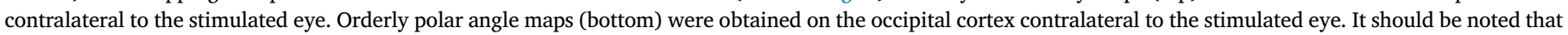
the maps were obtained with the mirror-pRF model across VM for FHONDA and with the single-pRF model for the control, as detailed in Methods and Results.

$53.16 \% \pm 0.35$, (iv) $52.4 \% \pm 0.32$; and F2: (i) $58.44 \% \pm 0.24$, (ii) $59.15 \% \pm 0.223$, (iii) $51.43 \% \pm 0.21$, (iv) $48.76 \pm 0.21$ respectively]. While the single-pRF model was superior in the controls for both stimulation conditions, i.e. with central and jittered fixation, an adequate reference to the FHONDA data affected by nystagmus, [mean VE \pm SEM for the above mentioned models (i-iv) in $\mathrm{C} 1$ and $\mathrm{C} 2$ under central and (jittered) fixation conditions; C1: (i) $57.01 \% \pm 0.18$ (46.69\% \pm 0.15 ), (ii) $43.58 \% \pm 0.16 \quad(37.91 \% \pm 0.1182), \quad$ (iii) $\quad 30.97 \% \pm 0.17$ (33.43\% \pm 0.13$), \quad$ (iv) $46.27 \% \pm 0.19 \quad(39.8 \% \pm 0.16)$; and C2: $62.23 \% \pm 0.21 \quad(60.31 \% \pm 0.22), \quad 50.99 \% \pm 0.18 \quad(49.96 \% \pm 0.18)$, $38.94 \% \pm 0.18(38.37 \% \pm 0.17), 48.95 \% \pm 0.20(47.86 \% \pm 0.19)]$. The relationship between pRF size and eccentricity, depicted in Fig. 2D, was another evidence confirming the superiority of the mirror-pRF model across VM in FHONDA. Specifically, the dependence of pRF sizes on eccentricity yielded systematically smaller receptive fields for the mirrorpRF model. It is plausible that this effect is due to increased pRF size estimates, if a single-pRF model has to cover mirror-symmetrical visual field locations in a single pRF (Hoffmann et al., 2012). Moreover, the overall small difference in variance explained between the mirror-pRF model across VM and single-pRF model in FHONA observed in Fig. 2C may be due to the larger pRF sizes of the single model. In order to test this assumption, the two models were compared with identical parameters. Initially, a mirror-pRF model across VM was fitted to fMRI time series. Afterwards, the pRF parameters (center positions and size) of this model were extracted and fed to a single-pRF model. The comparison of the mirror-pRF model across VM with its identical single counterpart confirmed the outperformance of the former model in both FHONDA participants [mean $\mathrm{VE} \pm \mathrm{SEM}$ for the above models in F1: $60.39 \% \pm 0.3053$ vs $52.77 \% \pm 0.3027$ and $\mathrm{F} 2: 59.13 \% \pm 0.2275$ vs $54.43 \% \pm 0.2675$; see suppl Fig. 3]. Additionally, a single-pRF model was fitted to the time series. Next, a mirror-pRF model across VM was obtained by taking the parameters of the single model and adding a mirror-symmetrical field. The comparison of these two models revealed only a negligible drop in the variance explained by the mirror-pRF model across VM [mean VE \pm SEM for the two models in F1: $58.85 \% \pm 0.3090$ vs $58.44 \% \pm 0.2990$ and $\mathrm{F} 2: 58.41 \% \pm 0.2426$ vs $57.18 \% \pm 0.2284$; see suppl Fig. 3]. Taken together, the mirrored-pRF model across VM clearly outperforms the single pRF-model in FHONDA for relatively small pRFs.

It must be noted that the cortical mapping observed in F2 (suppl. Fig. 1B) suggested the symmetry axis to be shifted eccentrically by $1.5^{\circ}$, instead of coinciding with the central vertical meridian, possibly as a result of eccentric fixation due to the patient's foveal hypoplasia; this shift was compensated for in the analyses of F2 depicted in Figs. 2-4. As a sanity check, we applied the same shift of the symmetry axis in controls and recomputed the pRF models; importantly the conventional singlepRF model still explained most of the variance in the control data, confirming that the shift of the symmetry axis was not the cause of the superiority of the mirror-pRF model in F2 (suppl. Fig. 2).

The above comparison of different pRF models demonstrated the mirror-pRF model across VM to be superior in FHONDA. This model was therefore applied for further analyses of the bilateral mapping data in FHONDA to assess the topography of the cortical visual field representation. The resulting eccentricity and polar angle maps in the early visual cortex are depicted in Fig. 3, where they are juxtaposed to control data (C1; for $\mathrm{C} 2$ see suppl. Fig. 1A) analysed with the more adequate singlepRF model for controls. These maps had a number of important features. Orderly eccentricity and polar angle maps, allowing for the delineation of the early visual areas, were obtained in both FHONDA and controls. Critically, in both FHONDA patients these maps were, in contrast to the controls, confined to the hemisphere contralateral to the stimulated eye.

From the above findings, we concluded that opposing hemifields in the two FHONDA participants were represented as a cortical overlay of mirror-symmetrical visual field positions in the hemisphere contralateral to the stimulated eye. This was directly tested with an analysis of the data-sets obtained during pRF-mapping of each hemifield in separate experiments. It should be mentioned that, as here only one hemifield was stimulated per experiment, even in FHONDA, the voxels should be driven only by a single pRF, i.e. in the stimulated visual hemifield. Consequently, the single-pRF model was applied as the most adequate approach to capture the response characteristics. In this analysis orderly eccentricity and polar angle maps (allowing for the same delineation of the early visual areas as in Fig. 3) were obtained (see Fig. 4). As for bilateral pRF-mapping, the maps in FHONDA were confined to the hemisphere contralateral to the stimulated eye. These features were in accordance with the conclusions drawn from the bilateral pRF-mapping data, i.e. the superposition of mirror-symmetrical visual field locations in the early visual cortex.

\section{Discussion}

In the current study, the first evidence on the nature of the 
A) Right hemifield
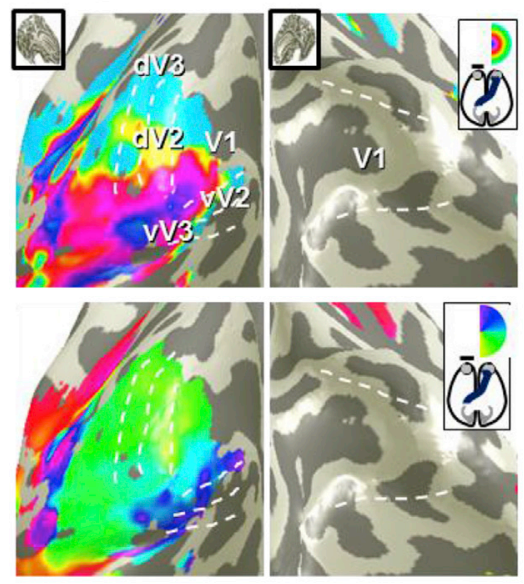

B) Left hemifield
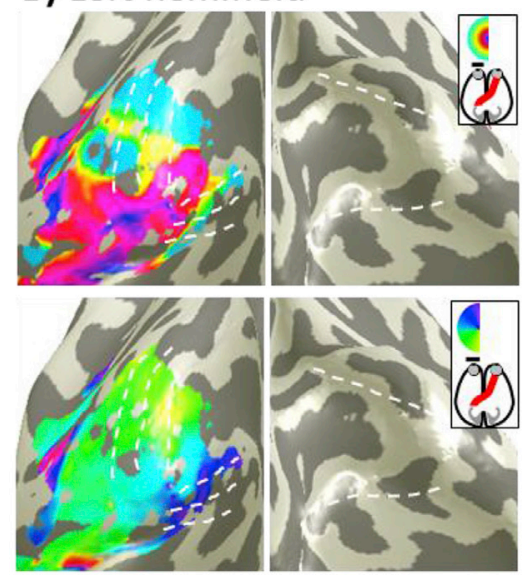

F1
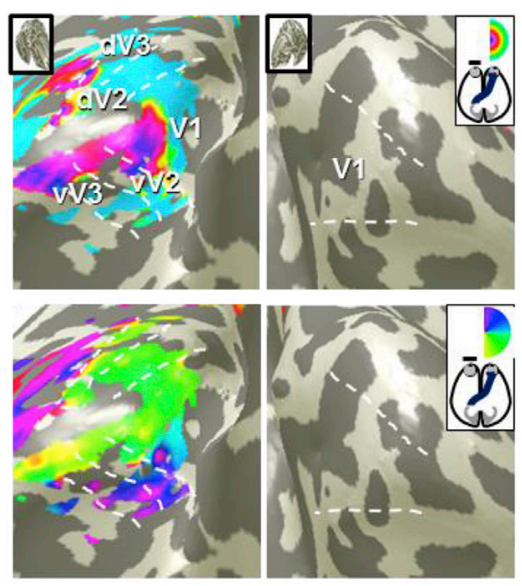

F2
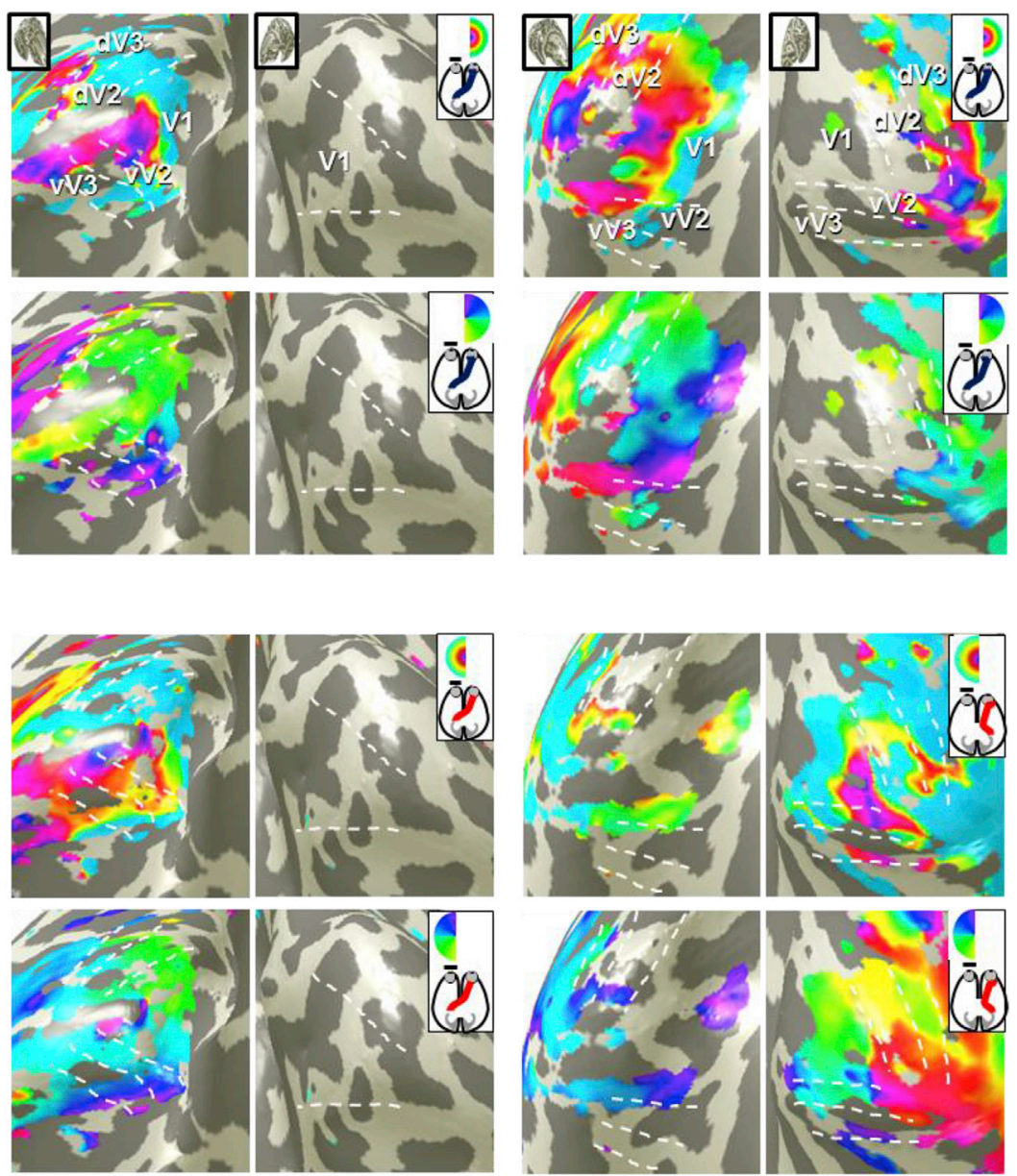

C1
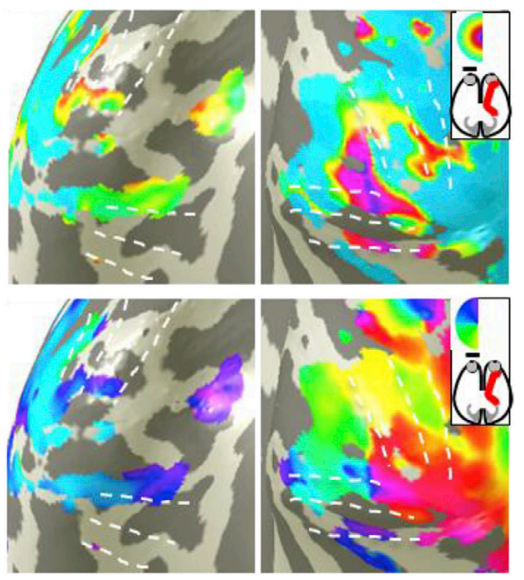

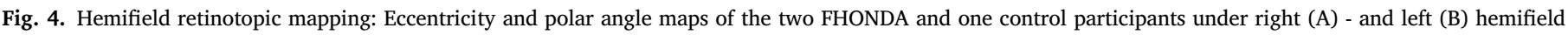

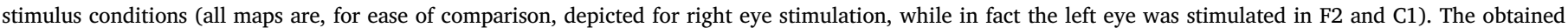

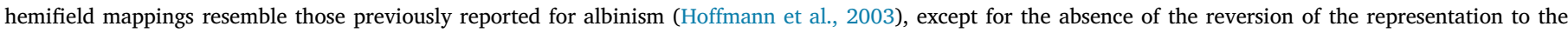

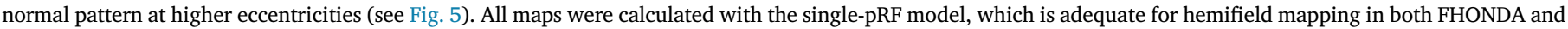

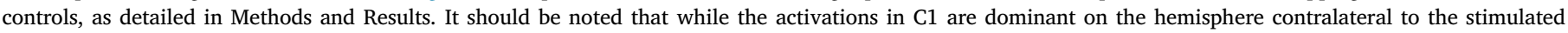

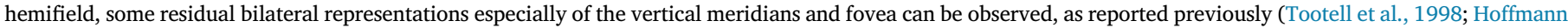
et al., 2003).

organization of the visual cortex in FHONDA is presented. Using both bilateral and hemifield stimulation, we report (i) the cortical responses to be confined to the hemisphere contralateral to the stimulated eye and (ii) the abnormal temporal retinal input to the visual cortex to be organized as a retinotopic map of the ipsilateral visual field that is superimposed onto the normal retinotopic map of the contralateral field. Consequently, visual field locations that are mirror-symmetric across the vertical meridian are co-localised in the visual cortex.

\section{Comparison to other congenital chiasmatic malformations}

The mapping observed in FHONDA is consistent with a conservative geniculo-striate projection, which is claimed for all other congenital chiasmatic malformations that were investigated in sufficient detail in humans, i.e. albinism, achiasma, and hemihydranencephaly (Hoffmann \& Dumoulin, 2015). This projection is taken as evidence for limited plasticity of the geniculo-striate projections in the face of abnormal input to the lateral geniculate nucleus (LGN). Normally, the LGN receives binocular input, which is projected in an interdigitating manner to the primary visual cortex to form ocular dominance domains. In the above chiasmatic malformation, the LGN receives abnormal monocular input from both hemifields, which appears to be projected via largely unchanged geniculo-striate connections in an interdigitating manner to the primary visual cortex, thus giving rise to hemifield domains (Guillery et al., 1984; Olman et al., 2016), which at lower resolutions show as superimposed hemifield maps (Hoffmann \& Dumoulin, 2015). The latter are reported here for FHONDA. Due to the uniformity of this projection across the range of congenital chiasmatic malformation it is concluded that the limited plasticity of the geniculo-striate projection is a general feature of human visual pathway formation, while more sub-cortical plasticity is available in some animal models (reviewed in Hoffmann et al., 2003).

\section{Distinction of FHONDA and albinism}

While FHONDA and albinism share important features, i.e. a hypoplastic fovea and enhanced optic nerve crossing, there is a profound difference. FHONDA is entirely independent of pigmentation deficits, whereas albinism is per definition associated with a pigmentation deficit, caused by reduced or even absent melanin synthesis. This is of particular importance, as the mechanism leading to the optic nerve misrouting in albinism is tightly linked to the reduced synthesis of melanin evident in albinism. Current evidence strongly supports the following view: retinal levels of L-DOPA, a melanin precursor, are reduced during embryonic 
A) Control

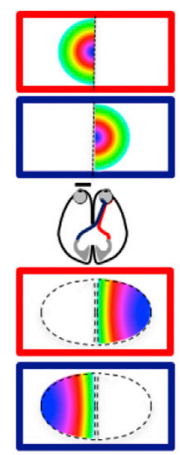

B) Albinism

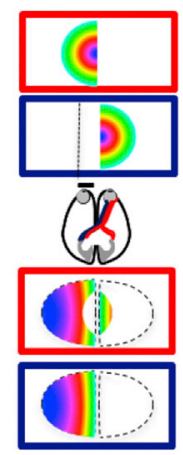

C) FHONDA

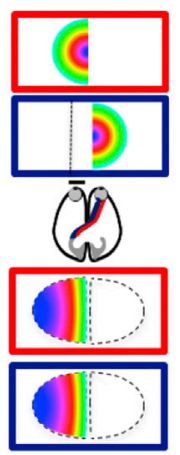

Visual field

Visual pathway

V1 representations
Fig. 5. Schematic optic nerve projections and eccentricity maps in V1 for (A) controls, (B) albinism, and (C) FHONDA: A) Control. Normally the nasal (blue) and temporal (red) retinal fibers of the right eye project to the left and right hemisphere (middle), respectively. As a result the eccentricity representations (false colour-coded) reside in the V1 contralateral to the respective hemifield. B) Albinism. Due to an enhanced crossing of the temporal retinal fibers to the contralateral hemisphere the line of decussation (dotted line) is shifted into the temporal retina. This results in the cortical overlay of orderly eccentricity maps from the contra- and ipsilateral hemifield for the part of the temporal retina that projects contralaterally, i.e. up to the line of decussation. Beyond the line of decussation, the representation reverts due to the ipsilateral projection from the temporal retina to the normal pattern, i.e. a representation of visual field on the contralateral hemisphere. C) FHONDA. The crossing of the temporal retinal fibers to the contralateral hemisphere results in the cortical overlay of orderly eccentricity maps from the contra- and ipsilateral hemifield for the entire temporal retina stimulated. Unlike albinism, no normal residual ipsilateral representation is observed, which suggests that the entire temporal retinal afferents might project to the contralateral hemisphere. development in albinism. This delays the differentiation of the ganglion cells, which as a consequence miss the time-window for development of the uncrossed projection at the optic chiasm (Ilia \& Jeffery, 1999; Jeffery, 2001; Hoffmann \& Dumoulin, 2015). The strong link between misrouting and reduced melanin synthesis in human albinism is underlined by the correlation of the pigment deficit and the extent of misrouting (von dem Hagen et al., 2007). In fact the degree of misrouting, i.e. the eccentricity up to which the abnormal projection prevails, varies between $2^{\circ}$ and $15^{\circ}$ degrees (Hoffmann et al., 2005), with the normal representation already 'fading in' prior to these values (Hoffmann et al., 2003). In FHONDA there is no indication of any pigmentation deficit, suggesting that the cause of misrouting is independent of melanin-synthesis and also of L-DOPA levels. Direct evidence for this notion, e.g. from pharmacological studies in animal models of FHONDA, is still pending. However, it should be noted that we did not observe any indication of a normal representation of the temporal retina in FHONDA, as it would be typical for albinism (see Fig. 5). This might, within the limits of stimulus dimensions in the current study ( $6^{\circ}$ stimulus radius), indicate that the underlying mechanism simply blocks uncrossed projections at the optic chiasm entirely. This contrasts with the partial and variable misrouting in albinism and is therefore suggestive of mechanisms in FHONDA and albinism that influence different molecular cascades, possibly operating at different sites. In conclusion, further investigations are necessary to test the notion that the mechanism underlying the decussation error in FHONDA is distinct from that albinism, i.e. from reduced retinal L-DOPA levels.

\section{Relevance for perception}

As for albinism and achiasma, the FHONDA participants of the current study had reductions of visual acuity, nystagmus and reduced binocular visual function, including strabismus and stereo-blindness. Reduced visual acuity is related to foveal hypoplasia and nystagmus, whereas stereo-blindness is a likely consequence of the misrouting of the optic nerves itself, disturbing the communication of the input from the two eyes to the visual cortex. As for albinism and achiasma, it appears that in FHONDA the disruption of the normal visual field maps by misrouting does not cause specific visual field defects and leaves major aspects of visual processing virtually intact. Qualitatively, this is evident from the observation that affected individuals make effective use of their vision in daily life, including reading. In albinism and achiasma specific investigations on the independence of the perception in opposing hemifields, indicate that mechanisms of cortical plasticity provide sufficient scope to support independent processing of the superimposed hemifields (Klemen et al., 2012; Bao et al., 2015, respectively). While such investigations are missing for FHONDA, the every-day behaviour of the two study participants does not reveal specific confusions of the left and right hemifields in visual perception. It is therefore assumed for FHONDA that the abnormal representation is made available for relatively normal visual perception via a similar interplay of subcortical stability and cortical plasticity as that inferred for the other types of chiasmatic malformations.

\section{Importance for clinical applications and for basic neuroscience}

Our fMRI-results confirm and extend previous electrophysiological indications of misrouted optic nerves in FHONDA, a syndrome clearly distinct from albinism as the melanin-synthesis is entirely unaffected. The account of enhanced optic-nerve crossing in FHONDA is of particular clinical importance, as this type of misrouting can no longer be regarded pathognomonic for albinism. Unclear cases of albinism, e.g. with mild hypopigmentation, must therefore not only be guided by the detection of misrouting, e.g. via visual evoked potentials, but need to be seconded by other features such as subtle pigmentation deficits as evidenced by iris translucency. Given the small number of FHONDA patients identified up to now, the diagnostic problem might be viewed as of little relevance. However, it must be noted that the actual number of affected individuals might be much higher, as FHONDA is a novel entity, and the number of previously undetected or misdiagnosed individuals is at present unclear.

In summary, our direct evidence of abnormal mapping in FHONDA highlights the importance of melanin-synthesis independent components required for the development of the human visual system, specifically the optic chiasm. Further the similarity of the cortical organization in FHONDA and other congenital visual pathway disorders indicates that interplay of stable geniculo-striate connections with intra-cortical plasticity is fundamental to the development of human visual system.

\section{Acknowledgements}

This work was supported by European Union's Horizon 2020 research and innovation programme under the Marie Sklodowska-Curie grant agreement (No 641805) to M.B.H and S.O.D. and the German Research Foundation (DFG: HO2002/10-2) to M.B.H. The authors are grateful for cooperation of the participants and the insightful feedback from the reviewers on an earlier version of the manuscript.

\section{Appendix A. Supplementary data}

Supplementary data related to this article can be found at https://doi. org/10.1016/j.neuroimage.2018.02.053. 


\section{References}

Al-Araimi, M., Pal, B., Poulter, J.A., van Genderen, M.M., Carr, I., Cudrnak, T., Toomes, C., 2013. A new recessively inherited disorder composed of foveal hypoplasia, optic nerve decussation defects and anterior segment dysgenesis maps to chromosome 16q23.3-24.1. Molecular Vision 19, 2165-2172.

Apkarian, P., Reits, D., Spekreijse, H., Van Dorp, D., 1983. A decisive electrophysiological test for human albinism. Electroencephalography and Clinical Neurophysiology 55 (5), 513-531.

Apkarian, P., Bour, L., Barth, P.G., 1994. A unique achiasmatic anomaly detected in nonalbinos with misrouted retinal-fugal projections. The European Journal of Neuroscience 6 (3), 501-507.

Bao, P., Purington, C.J., Tjan, B.S., 2015. Using an achiasmic human visual system to quantify the relationship between the fMRI BOLD signal and neural response. ELife 4 e09600. https://doi.org/10.7554/eLife.09600.

Brainard, D.H., 1997. The psychophysics toolbox. Spatial Vision 10 (4), 433-436.

Davies-Thompson, J., Scheel, M., Lanyon, L.J., Barton, J.J.S., 2013. Functional organisation of visual pathways in a patient with no optic chiasm. Neuropsychologia 51 (7), 1260-1272. https://doi.org/10.1016/j.neuropsychologia.2013.03.014.

Dumoulin, S.O., Wandell, B.A., 2008. Population receptive field estimates in human visual cortex. NeuroImage 39 (2), 647-660. https://doi.org/10.1016/j.neuroimage. 2007.09.034.

Fracasso, A., Koenraads, Y., Porro, G.L., Dumoulin, S.O., 2016. Bilateral population receptive fields in congenital hemihydranencephaly. Ophthalmic and Physiological Optics 36 (3), 324-334. https://doi.org/10.1111/opo.12294.

Friston, K.J., Fletcher, P., Josephs, O., Holmes, A., Rugg, M.D., Turner, R., 1998. Eventrelated fMRI: characterizing differential responses. NeuroImage 7 (1), 30-40. https:// doi.org/10.1006/nimg.1997.0306.

Guillery, R.W., Hickey, T.L., Kaas, J.H., Felleman, D.J., Debruyn, E.J., Sparks, D.L., 1984 Abnormal central visual pathways in the brain of an albino green monkey (Cercopithecus aethiops). The Journal of Comparative Neurology 226 (2), 165-183. https://doi.org/10.1002/cne.902260203.

Harvey, B.M., Dumoulin, S.O., 2011. The relationship between cortical magnification factor and population receptive field size in human visual cortex: constancies in cortical architecture. The Journal of Neuroscience: The Official Journal of the Society for Neuroscience 31 (38), 13604-13612. https://doi.org/10.1523/JNEUROSCI. 2572-11.2011.

Hoffmann, M.B., Tolhurst, D.J., Moore, A.T., Morland, A.B., 2003. Organization of the visual cortex in human albinism. The Journal of Neuroscience: The Official Journal of the Society for Neuroscience 23 (26), 8921-8930.

Hoffmann, M.B., Lorenz, B., Morland, A.B., Schmidtborn, L.C., 2005. Misrouting of the optic nerves in albinism: estimation of the extent with visual evoked potentials. Investigative Ophthalmology \& Visual Science 46 (10), 3892-3898. https://doi.org/ 10.1167/iovs.05-0491.

Hoffmann, M.B., Seufert, P.S., Schmidtborn, L.C., 2007. Perceptual relevance of abnorma visual field representations: static visual field perimetry in human albinism. The British Journal of Ophthalmology 91 (4), 509-513. https://doi.org/10.1136/bjo. 2006.094854.

Hoffmann, M.B., Kaule, F.R., Levin, N., Masuda, Y., Kumar, A., Gottlob, I., Dumoulin, S.O., 2012. Plasticity and stability of the visual system in human achiasma. Neuron 75 (3), 393-401. https://doi.org/10.1016/j.neuron.2012.05.026.
Hoffmann, M.B., Dumoulin, S.O., 2015. Congenital visual pathway abnormalities: a window onto cortical stability and plasticity. Trends in Neurosciences 38 (1), 55-65. https://doi.org/10.1016/j.tins.2014.09.005.

Ilia, M., Jeffery, G., 1999. Retinal mitosis is regulated by dopa, a melanin precursor that may influence the time at which cells exit the cell cycle: analysis of patterns of cell production in pigmented and albino retinae. The Journal of Comparative Neurology 405 (3), 394-405.

Jeffery, G., 2001. Architecture of the optic chiasm and the mechanisms that sculpt its development. Physiological Reviews 81 (4), 1393-1414.

Kaule, F.R., Wolynski, B., Gottlob, I., Stadler, J., Speck, O., Kanowski, M., Hoffmann, M.B., 2014. Impact of chiasma opticum malformations on the organization of the human ventral visual cortex. Human Brain Mapping 35 (10), 5093-5105. https://doi.org/10.1002/hbm.22534.

Klemen, J., Hoffmann, M.B., Chambers, C.D., 2012. Cortical plasticity in the face of congenitally altered input into V1. Cortex. J. Devoted to the Study of the Nervous System and Behavior 48 (10), 1362-1365. https://doi.org/10.1016/j.cortex.2012. 03.012 .

Muckli, L., Naumer, M.J., Singer, W., 2009. Bilateral visual field maps in a patient with only one hemisphere. Proceedings of the National Academy of Sciences 106 (31), 13034-13039. https://doi.org/10.1073/pnas.0809688106.

Olman, C.A., Bao, P., Engel, S.A., Grant, A.N., Purington, C., Qiu, C., Tjan, B.S., 2016. Hemifield columns co-opt ocular dominance column structure in human achiasma. NeuroImage. https://doi.org/10.1016/j.neuroimage.2016.12.063.

Pelli, D.G., 1997. The VideoToolbox software for visual psychophysics: transforming numbers into movies. Spatial Vision 10 (4), 437-442.

Poulter, J.A., Al-Araimi, M., Conte, I., van Genderen, M.M., Sheridan, E., Carr, I.M., Toomes, C., 2013. Recessive mutations in SLC38A8 cause foveal hypoplasia and optic nerve misrouting without albinism. American Journal of Human Genetics 93 (6), 1143-1150. https://doi.org/10.1016/j.ajhg.2013.11.002.

Teo, P.C., Sapiro, G., Wandell, B.A., 1997. Creating connected representations of cortical gray matter for functional MRI visualization. IEEE Transactions on Medical Imaging 16 (6), 852-863. https://doi.org/10.1109/42.650881.

Tootell, R.B., Mendola, J.D., Hadjikhani, N.K., Liu, A.K., Dale, A.M., 1998. The representation of the ipsilateral visual field in human cerebral cortex. Proceedings of the National Academy of Sciences 95 (3), 818-824.

van Genderen, M.M., Riemslag, F.C.C., Schuil, J., Hoeben, F.P., Stilma, J.S., Meire, F.M., 2006. Chiasmal misrouting and foveal hypoplasia without albinism. The British Journal of Ophthalmology 90 (9), 1098-1102. https://doi.org/10.1136/bjo.2006. 091702 .

Victor, J.D., Apkarian, P., Hirsch, J., Conte, M.M., Packard, M., Relkin, N.R., Shapley, R.M., 2000. Visual function and brain organization in non-decussating retinal-fugal fibre syndrome. Cerebral Cortex 10 (1), 2-22 (New York, N.Y.: 1991)

von dem Hagen, E.A.H., Houston, G.C., Hoffmann, M.B., Morland, A.B., 2007. Pigmentation predicts the shift in the line of decussation in humans with albinism. The European Journal of Neuroscience 25 (2), 503-511. https://doi.org/10.1111/j. 1460-9568.2007.05303.x.

von dem Hagen, E.A.H., Hoffmann, M.B., Morland, A.B., 2008. Identifying human albinism: a comparison of VEP and fMRI. Investigative Ophthalmology \& Visual Science 49 (1), 238-249. https://doi.org/10.1167/iovs.07-0458. 